\title{
Therapy of colon carcinoma: An oncology perspective
}

\author{
Piotr M Czaykowski MD MSc FRCPC ${ }^{1}$, Paul C Adams MD FRCPC ${ }^{2}$, Editor-in-Chief
}

$\mathrm{D}_{\mathrm{c}}^{\mathrm{r}}$ Piotr Czaykowski is an Assistant Professor and Medical Oncologist, Department of Medical Oncology and Haematology, CancerCare Manitoba, Winnipeg. PA: As gastroenterologists we are often the physicians that make the diagnosis of colon cancer...It seems that decisions about postoperative therapy are based on this assessment. Is this ideal at the present time?

PC: There is no question that a medical oncologist's recommendation regarding the need for systemic therapy is heavily dependent on the staging information gleaned in the initial workup. We look for the following crucial elements: Is the cancer localized? If localized, is it rectal or colon cancer? If localized, what is the status of the parameters that are linked with the risk of recurrence after surgery? If not localized, what is the extent of metastatic disease?

Most commonly, the first investigation is an endoscopic evaluation of the cancer, and frequently this is performed by a gastroenterologist. This not only allows for confirmation of malignancy, but also proves crucial in helping medical and radiation oncologists determine the need for radiotherapy in distal large bowel tumours.

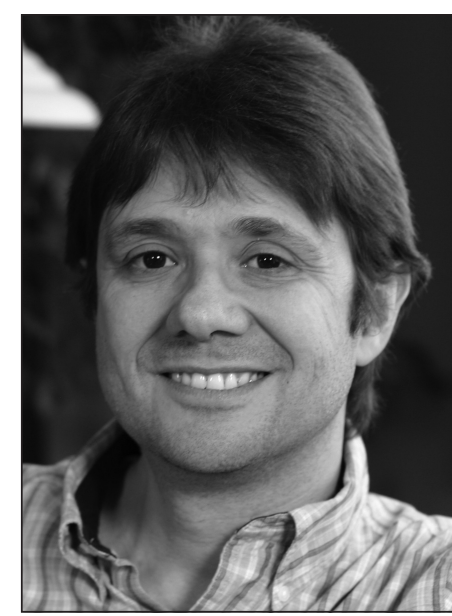

Dr Piotr Czaykowski is an Assistant Professor and Medical Oncologist, Department of Medical Oncology and Haematology, CancerCare Manitoba, Winnipeg tomography scan of the abdomen and pelvis. A preoperative carcinoembryonic antigen test (CEA) is helpful, primarily as a prognostic indicator (2). Because I am focusing on colon cancer, I will not address the role of magnetic resonance imaging and endoscopic ultrasound in staging rectal cancer.

When the patient does undergo surgery, generally for apparently localized disease, it is important that the surgeon confirm that there are no obvious metastases (and reports on this), and that he or she focuses on harvesting an adequate number of lymph nodes. It has been recognized for some time that insufficient nodal sampling leads to under-staging; the exact number of nodes necessary for accurate nodal staging remains controversial but many centres use 12 as the magic number. Clearly, finding an adequate number of nodes depends to some extent on the surgeon, but it is also heavily reliant on the pathology team. Even if positive nodes are identified, it appears to be important to remove as many as possible, because there are now some data that suggest the number and ratio of positive nodes are also important in prognosis $(3,4)$.

Currently, there are no recommendations Although the definition of where the rectum ends and the sigmoid begins remains controversial, clinical trials commonly define the rectum as being the distal $12 \mathrm{~cm}$ to $15 \mathrm{~cm}$ of the large bowel, and most of us use that as our day-to-day reference point. If I start cursing in clinic, it is usually because I have an endoscopic report that says rectal cancer (without any measurement), an operative note that calls the tumor rectosigmoid, and a computed tomography scan that calls it sigmoid. Because we only use radiotherapy routinely for rectal cancers, this question of location becomes an important one - so an explicit statement by the endoscopist of the location of the tumour is of great consequence. It is also helpful in advanced cancers to have some indication of the patency of the lumen.

Even in this day and age, a surprising number of patients undergo a primary bowel resection without adequate staging. This leads to some unnecessary surprises, and occasionally leads to poor intraoperative choices. There appears to be an emerging consensus that patients with extensive metastatic disease with relatively asymptomatic primary tumours are probably best served by commencing chemotherapy as soon as possible, and worrying about the primary tumour later, if symptoms necessitate (1). Thus, the key staging investigations, often initiated by the gastroenterologist, ideally should be done before resection of the primary tumour, and should include chest imaging and a computed for the routine use of positron emission tomography scanning in apparently localized colon cancer. Those centres that have ready access most commonly use this modality for trying to determine if a patient with metastatic disease can be considered for resection of the metastases with curative intent. Similarly, the use of other tumour markers (for example CA 19-9) and the use of molecular profiling (eg, looking for microsatellite instability or for overexpression of vascular endothelial growth factor or epidermal growth factor) remains the domain of clinical trials for the most part.

A problem across most of Canada is that there are often excessive delays in getting the patient through the whole process of diagnosis and treatment. In a recent review of 93 Manitoba patients with stage III colon cancer who received adjuvant chemotherapy, we found that of the 58 identified as having colon cancer before surgery, the median time from diagnosis to surgery was 24 days, with $40 \%$ of patients waiting more than four weeks. After surgery, the median wait to start adjuvant chemotherapy was 55 days, with approximately one-third of this time reflecting a delay in referral to medical oncology.

PA: Can you update us on the optimal oncology treatments for colon cancer in Canada?

PC: Although there are some minor variations among provinces, most seem to adhere to very similar standards. Because

\footnotetext{
${ }^{1}$ Departments of Internal Medicine (Section of Haematology/Oncology) and Community Health Sciences, University of Manitoba; Department of

Medical Oncology and Haematology, CancerCare Manitoba, Winnipeg, Manitoba; ${ }^{2}$ University Hospital, London, Ontario

Correspondence: Dr Piotr M Czaykowski, CancerCare Manitoba, 675 McDermot Avenue, Winnipeg, Manitoba R3E OV9.

Telephone 204-787-1992, fax 204-786-0196, e-mail Piotr.Czaykowski@cancercare.mb.ca

Received for publication March 26, 2007. Accepted March 26, 2007
} 
most systemic therapies are tested first in advanced disease, before moving into the adjuvant setting, I will discuss advanced colorectal cancer (ACRC) first.

There is no question that the currently available systemic therapies have led to improvement in outcomes in ACRC. When I completed my training in the mid-1990s, the only drug readily available to us was 5 -fluorouracil (5-FU, often referred to as 5-flipping-useless, or words to that effect). Objective shrinkage of disease was seen in no more than $25 \%$ of patients and median survival was around 12 months. Since that time, the introduction of new conventional chemotherapy agents, particularly irinotecan and oxaliplatin, has led to substantial increases in both objective response rates and in survival. Additionally, changing the way we use 5-FU has led to improved toxicity profiles. The most common strategy now employed in Canada for ACRC is to start with a combination of 5-FU (by bolus injection and continuous infusion), leucovorin and either oxaliplatin or irinotecan (these commonly used regimens have the acronyms FOLFOX and FOLFIRI, respectively). At the time of disease progression, second-line treatment is instituted by maintaining the 5-FU and leucovorin and switching the oxaliplatin or irinotecan. This strategy is leading to median survivals in the range of 18 to 20 months, generally with a reasonable quality of life (5). All of us are seeing patients alive three years or more postdiagnosis of ACRC, a previously rare experience. This has led to new dilemmas, including whether we need to administer chemotherapy continuously as long as patients are stable, how to avoid cumulative toxicities (particularly peripheral neuropathy with oxaliplatin) and how we cope with the added workload of long-term patients coming for chemotherapy every two weeks for months to years. Additionally, we are now faced with the difficult issue of what to do for patients who are still well but have progressed on second-line chemotherapy.

There is increasing interest in substituting capecitabine (an oral analogue of 5-FU that is at least as effective as 5-FU and folinic acid [FUFA] and less toxic) for the infusional 5-FU/leucovorin in multi-drug regimens in the interest of simplicity and decreasing demand on chemotherapy suites. Preliminary results from recently completed studies suggest that this substitution does not compromise outcomes. There remains some interest in the role of raltitrexed, a drug that targets the same enzyme as 5-FU and is administered intravenously once every three weeks. Whereas 5-FU is known to cause coronary spasm, raltitrexed has not been found to cause this side effect, making it appealing for patients with underlying coronary artery disease. Raltitrexed is probably slightly less effective than FUFA, but has been tested in combination with oxaliplatin, with acceptable toxicity and reasonable efficacy.

There is considerable controversy in Canada about the role of the new monoclonal antibodies, bevacizumab and cetuximab, in the management of ACRC. These agents are very expensive, and the exact degree of additional benefit is not well defined. This will be discussed further below.

In patients with localized disease, adjuvant chemotherapy is generally recommended for all medically fit patients with stage III (node-positive) disease. For those with node-negative disease, with cancer extending into the pericolic adipose tissue (stage II), there is general agreement to offer adjuvant therapy to those considered to be at higher than average risk of recurrence. The factors clearly regarded as leading to increased risk are: T4 primary tumours (either invading onto the serosal surface or into adjacent organs), poorly differentiated histology, the presence of perforation and assessment of an inadequate number of nodes (see above). Other factors are thought to possibly increase the risk of cancer recurrence and may also influence the decision to offer adjuvant therapy. These include an obstructing primary tumour, lymphovascular invasion, perineural invasion and tumours with a high proliferative index. There is also a preference toward offering adjuvant therapy to young patients even if they have average-risk stage II disease. Adjuvant therapy is not offered after resection of stage I colon cancer.

The standard adjuvant chemotherapy offered is some version of the FOLFOX regimen, given every two weeks for 12 cycles. FOLFOX-4 has been demonstrated to decrease the risk of recurrence of stage III colon cancer by an absolute $6.3 \%$ at three years compared with infusional 5-FU and leucovorin (6). Most Canadian centres use a version of this, referred to as modified FOLFOX-6, which is simpler to administer. The previous standard North American regimen of bolus 5-FU and leucovorin (FUFA or Mayo regimen) for five consecutive days every four weeks for six cycles has essentially been abandoned because it is less effective and too toxic. Some patients are offered the option of adjuvant therapy with capecitabine. This spares the need for a central venous access device (needed for the FOLFOX and FOLFIRI regimens) and requires far fewer cancer clinic visits. Unfortunately, there is considerable disparity among the provinces regarding whether capecitabine is funded by the province. There is growing interest in substituting capecitabine for infusional 5-FU in combination with oxaliplatin. The clinical trials testing the effectiveness of this substitution have not yet matured.

PA: There is growing concern about the cost of chemotherapy in situations where it is not likely to be curative. There are some drugs that have been advocated for colon cancer that are in this category.

PC: Currently, systemic therapy has no potential for curing ACRC. A small minority of patients with paucimetastatic disease may have sufficient shrinkage of their disease to enable potentially curative resection of metastases, but in general terms, the goal of systemic therapy remains control of the cancer. When cure is not the goal, the competing considerations relate to the degree of benefit versus the impact on quality of life. Obviously, cost also becomes an issue.

Although the new chemotherapy agents are expensive ( $\$ 20,000$ to $\$ 30,000$ per six months of therapy), their cost is not out of keeping with what we are willing to pay for similarly effective agents in other malignancies, and provincial Ministries of Health have been willing accept these added costs. What is more of an issue is the addition of the new 'targeted therapies' to the standard armamentarium for ACRC. Part of the problem relates to the uncertainty of the degree of benefit they provide, particularly in the context of their cost. Two agents in particular are creating controversy in Canada.

Bevacizumab, a monoclonal antibody targeted against vascular endothelial growth factor, has been demonstrated to prolong survival when added to chemotherapy. The most compelling study published to date (7) concluded that adding bevacizumab to a specific combination of 5-FU, leucovorin and irinotecan (IFL) led to a five month increase in median survival over IFL alone. This is considered a fairly dramatic result for an advanced solid tumour. However, in absolute terms, the combination of IFL plus bevacizumab generated a median survival of 20 months, which is no better than the results we see using regimens such as FOLFOX and FOLFIRI alone. IFL is considered to be a toxic 
chemotherapy regimen and is not in common use in Canada, so some have concluded that this 'seminal' study has done no more than demonstrate that adding bevacizumab to inferior chemotherapy yields results as good as better chemotherapy. A more recent study, so far only presented in abstract form, has suggested that bevacizumab does improve survival when added to the more effective and better tolerated FOLFOX chemotherapy, but with a more modest gain of approximately two month extension of median survival. A five month survival gain, at an incremental cost in Canada of approximately $\$ 50,000$ per patient per annum, is one thing, but if the actual benefit is only two months, one has to wonder if this is the best use of our resources. Although generally well tolerated, bevacizumab can lead to some serious toxicities including hypertension, thromboembolic events and bowel perforation, to name a few. This agent is currently being funded by a few provinces. In other provinces the governments have shut their eyes to its use in private infusion clinics, or have even permitted provincial cancer agencies to administer it to patients who have been able to purchase it on their own. One can hear the creaking of the Canada Health Act. There is also the issue that this agent has been aggressively marketed, with patients' perceptions of the gains often appearing unrealistic.

The second agent that is causing controversy is cetuximab (the drug that Martha Stewart went to jail over). This monoclonal antibody targets the epidermal growth factor receptor. Although licensed in Canada, it is not actually being marketed here because of a disagreement between the manufacturer and federal regulators over its price. Cetuximab has been positioned as a third-line agent, with studies indicating that it can reverse irinotecan resistance and prolong the time to cancer progression (8). However, there are still no published studies demonstrating a significant improvement in survival duration with this agent. A recently completed Canadian-led study looking at cetuximab alone as third-line treatment has been reported as meeting its primary end point, improved survival, compared with best supportive care. The first presentation of these results is expected soon. To the best of my knowledge this agent is not currently being used in Canada outside of clinical trials. In the United States, cetuximab is being priced at around $\$ 10,000$ per patient per month.

The Journal of Clinical Oncology, the most highly regarded oncology journal, recently devoted an entire issue to the burgeoning costs of cancer care. ACRC was a focus of several of the articles in the issue because of the spiraling costs. One article identified that, in the United States, the total drug costs for the management of metastatic colorectal cancer has risen from less than $\$ 1,000$ per patient (with a survival of approximately one year), to approximately $\$ 180,000$ per patient (with a survival of approximately two years) (9). Another author quotes a conversation with a pharmaceutical company executive as follows: "we don't have any cancer products in our pipeline that will be priced at less than $\$ 300,000$ per quality-adjusted life-year" (10).

In general, it appears that new targeted therapies are being priced in the range of $\$ 5,000$ to $\$ 10,000$ per patient per month (including new oral agents such as sunitinib and sorafenib, currently used in renal carcinoma). So far, none of these new agents have been demonstrated to cure advanced cancers, although some clearly extend survival. If we move into the realm of sequential or combination targeted therapies, usually in conjunction with chemotherapy at some point in the mix, we can anticipate costs per patient per annum in excess of $\$ 100,000$.
For the patient desperate to live long enough to see her child graduate from high school, this does not seem unreasonable. For provincial governments, it may appear otherwise. This will undoubtedly put to the test the notion of provincial equality to access of care, and the growth of the private sector in oncological care increasingly appears inevitable in Canada.

PA: In many types of cancer, we are moving toward a multimodality approach of chemotherapy, radiotherapy and surgery. Is this appropriate in colon cancer?

PC: There is already a well-established multimodal approach in stage II and stage III rectal cancer. These cancers are treated with surgery, chemotherapy and radiotherapy, although the optimal order of the treatments remains hotly debated. The options include a short course of 'hot' radiotherapy followed quickly by surgery, and then potentially six months of chemotherapy, versus a long course of chemoradiotherapy followed by surgery and then chemotherapy, versus surgery upfront followed by chemotherapy and long-course chemoradiotherapy. Of key importance is obtaining accurate pretreatment staging - this requires either a rectal magnetic resonance imaging or a transrectal ultrasound, or both.

In colon cancer, radiotherapy is not generally part of the standard treatment algorithm for either localized or advanced disease. I have discussed above the need for adjuvant chemotherapy in stage III and high-risk stage II colon cancer after definitive surgery.

An area receiving increasing attention is the role of chemotherapy in attempting to 'cure' ACRC. The observation has been made that patients with initially unresectable metastases confined to the liver can be down-staged with chemotherapy to the point where curative intent surgery can be attempted (11). Such an approach requires an aggressive mind-set and close coordination between the medical oncologist and the hepatobiliary surgeon. Objectively, it appears to be a legitimate goal for a small minority of patients with ACRC, but the rewards can be great indeed.

There also remains controversy about the role of 'adjuvant chemotherapy' after potentially curative resection of metastatic colorectal cancer. Most medical oncologists appear prepared to offer such treatment to selected patients, despite a lack of compelling evidence. This is based on the fundamental assumption that adjuvant chemotherapy is an effective treatment when there is at most micrometastatic disease left, and it should not really make a difference how you get to the point of having only micrometastatic disease.

PA: What do you see on the horizon as new treatments for advanced colon cancer?

PC: At the moment, the horizon looks a bit cloudy. I am not aware of any new agents that are displaying the promise that bevacizumab and cetuximab have held out to us over the last five years. I think there is little doubt that the future focus will remain on molecularly targeted therapies. We will increasingly view each cancer as unique, and attempt to target the aberrant pathways implicated in each individual cancer. Practical application of such a paradigm shift appears to be far off in the future - but in oncology, time seems to have a remarkable way of accelerating.

PA: In many cities, cancer care is quite separate from the general hospital. In patients with colon cancer there may be a gastroenterologist, surgeon and oncologist involved. When it comes to long-term follow-up, it seems to me that our routine polyp patients may be getting better follow-up than patients with previous cancer. 
PC: Point well taken. In general, medical oncologists are becoming increasingly busy, and have less time and inclination to follow the 'well' patient after completion of adjuvant chemotherapy. When we discharge patients we try to ensure that someone will be following them and we provide that individual with recommendations for follow-up. These recommendations tend to vary among centres and even among oncologists at the same centre. Increasingly, provincial cancer agencies are trying to generate formalized discharge guidelines and recommendations, but there remain big issues with who should follow the patient. For example, is this the role of the family physician? Our own experience is that they are frequently not very good at ensuring that patients are seen at the regular intervals we recommend. It is not uncommon for us to see a patient return with metastatic disease who has not had any routine follow-up after discharge on completion of their adjuvant therapy - despite the fact that we had written a letter to the family doctor outlining our recommendations. At CancerCare Manitoba, we are currently exploring ways of making this 'hand-over' more effective, in part by including the patient in the follow-up planning process.

An area of follow-up that is often particularly suspect is the ongoing endoscopic surveillance of the remaining bowel. There are issues about who should be responsible for this (the gastroenterologist who diagnosed the cancer? The surgeon who resected it?), and who should ensure that it is being conducted according to recommended time intervals (the person responsible for coordinating the overall follow-up plan? The gastroenterologist or surgeon who did the last scope?). So far, we have not satisfactorily addressed this deficiency in followup planning. At least in my province, there appear to be some overt political overtones to the entire debate.

\section{REFERENCES}

1. Stelzner S, Hellmich G, Koch R, Ludwig K. Factors predicting survival in stage IV colorectal carcinoma patients after palliative treatment: A multivariate analysis. J Surg Oncol 2005;89:211-7.

2. Park YJ, Park KJ, Park JG, Lee KU, Choe KJ, Kim JP. Prognostic factors in 2230 Korean colorectal cancer patients: Analysis of consecutively operated cases. World J Surg 1999;23:721-6.

3. LeVoyer TE, Sigurdson ER, Hanlon AL, et al. Colon cancer survival is associated with increasing number of lymph nodes analyzed: A secondary survey of intergroup trial INT-0089. J Clin Oncol 2003;21:2912-9.

4. Berger AC, Sigurdson ER, LeVoyer T, et al. Colon cancer survival is associated with decreasing ratio of metastatic to examined lymph nodes. J Clin Oncol 2005;23:8706-12.

5. Tournigand C, André T, Achille E, et al. FOLFIRI followed by FOLFOX6 or the reverse sequence in advanced colorectal cancer: A randomized GERCOR study. J Clin Oncol 2004;22:229-37.

6. André T, Boni C, Mounedji-Boudiaf L, et al. Oxaliplatin, fluorouracil, and leucovorin as adjuvant treatment for colon cancer. N Engl J Med 2004;350:2343-51.

7. Hurwitz H, Fehrenbacher L, Novotny W, et al. Bevacizumab plus irinotecan, fluorouracil, and leucovorin for metastatic colorectal cancer. N Engl J Med 2004;350:2335-42.

8. Cunningham D, Humblet Y, Siena S, et al. Cetuximab monotherapy and cetuximab plus irinotecan in irinotecan-refractory metastatic colorectal cancer. N Engl J Med 2004;351:337-45.

9. Meropol NJ, Schulman KA. Cost of cancer care: Issues and implications. J Clin Oncol 2007;25:180-6.

10. Ramsey SD. How should we pay the piper when he's calling the tune? On the long-term affordability of cancer care in the United States. J Clin Oncol 2007;25:175-9.

11. Poston G, Adam R, Vanthey JN. Downstaging or downsizing: Time for a new staging system in advanced colorectal cancer? J Clin Oncol 2006;24:2702-6. 


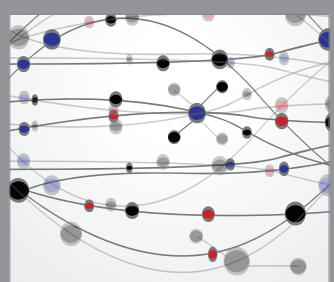

The Scientific World Journal
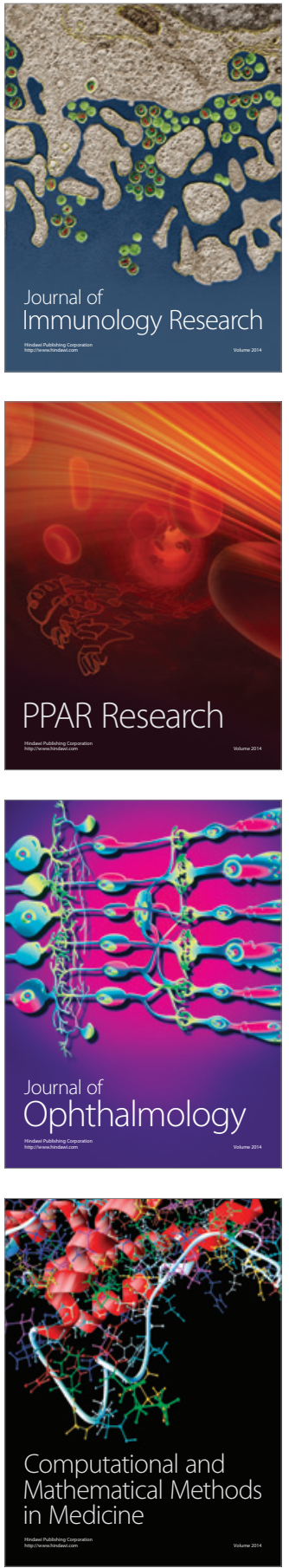

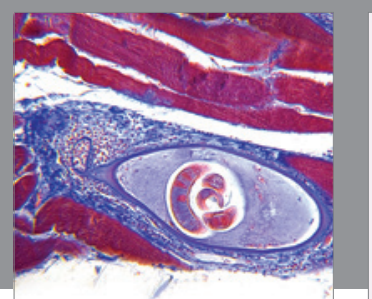

Gastroenterology Research and Practice

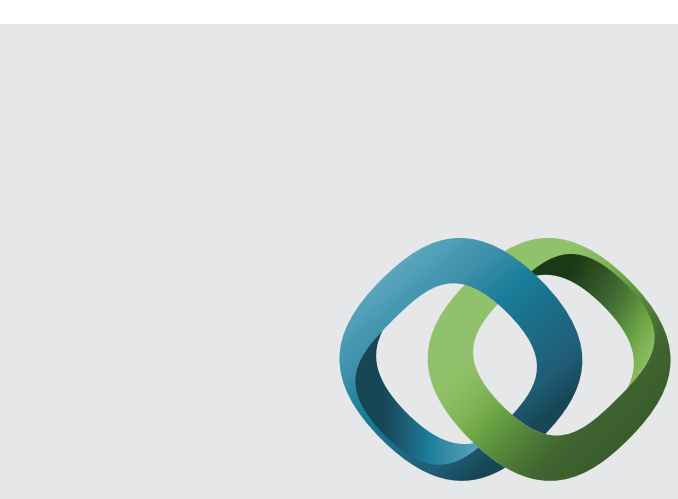

\section{Hindawi}

Submit your manuscripts at

http://www.hindawi.com
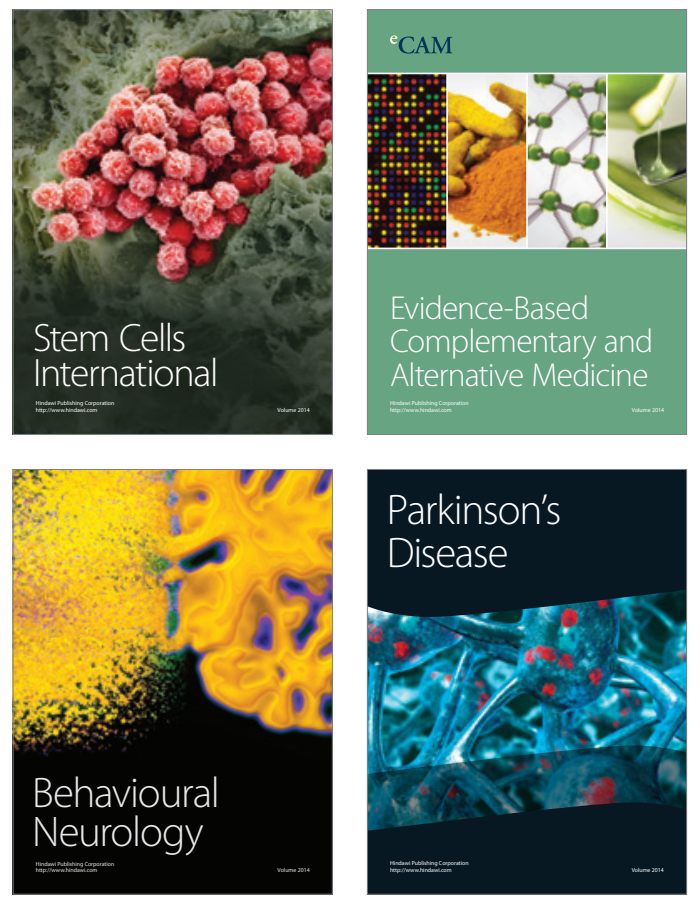
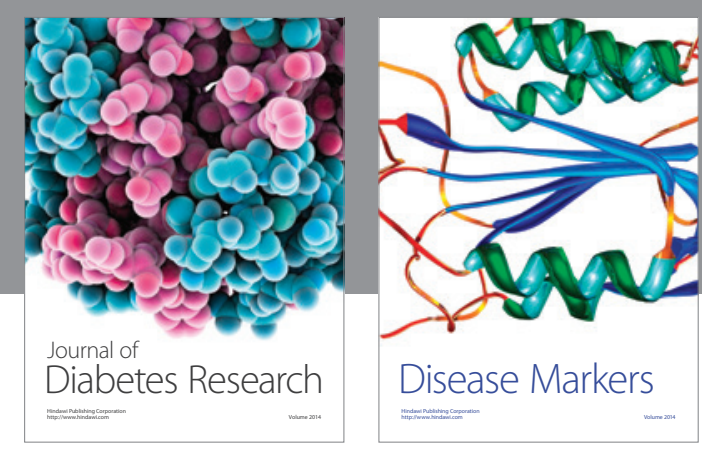

Disease Markers
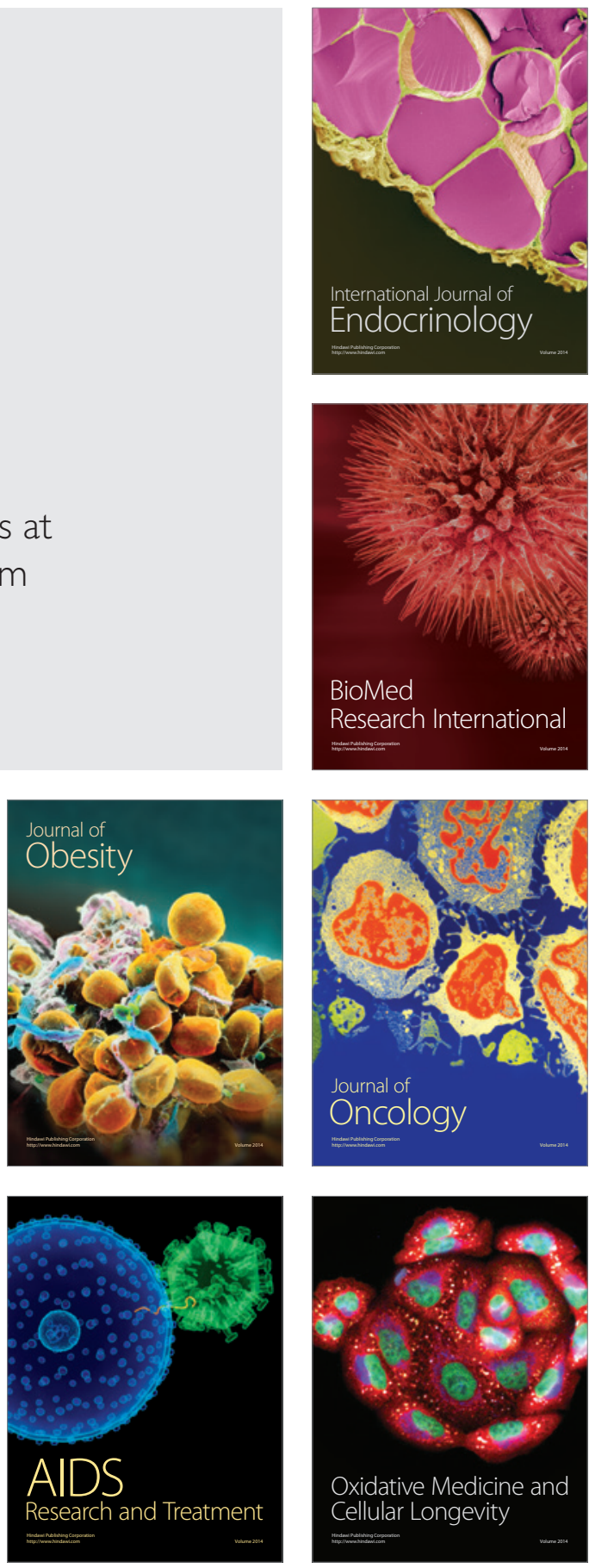